\title{
Seroprevalence against Borrelia burgdorferi sensu lato and occurence of antibody co-expression with Anaplasma phagocytophilum in dogs in Latvia
}

Inese Berzina* and Ilze Matise

\begin{abstract}
Background: Lyme disease is commonly diagnosed in humans in Latvia, but up to date no studies have been performed to investigate its prevalence in dogs. The aim of this study was to evaluate if seroprevalence against B. burgdorferi sensu lato (B. burgdorferi s.l.) and co-expression of antibodies against B.burgdorferi s.l. and A. phagocytophilum is higher in dogs with clinical suspicion of tick-borne diseases compared to healthy dogs.

Findings: Venous blood was taken from healthy dogs $(n=441)$ and dogs suspected to have borreliosis and/ or canine granulocytic anaplasmosis $(n=29)$. The presence of antibodies was detected with SNAP 4Dx test (IDEXX, Westbrook, Maine, USA). The seroprevalence against B. burgdorferi s.I. in healthy dogs was 2.49\% (11/441) and 36\% (4/11) of seropositive dogs had antibodies against both of investigated bacteria. None of the dogs in sick dog group had detectable antibodies against B. burgdorferi s.l.

Conclusions: We conclude that seroprevalence to $B$. burgdorferi s.l. in dogs in Latvia is low and that dogs with suspicion of tick-borne disease do not have higher $B$. burgdorferi s.l. seroprevalence than healthy dogs. Dogs that express antibodies against B. burgdorferi s.l. frequently co-express antibodies against A. phagocytophilum.
\end{abstract}

Keywords: B. burgdorferi sensu lato, A. phagocytophilum, Dogs, Seroprevalence, Latvia

\section{Findings}

Lyme disease and granulocytic anaplasmosis have been diagnosed in humans in Latvia (Bormane, [1]; Süss, [2]). Ixodes ricinus and $I$. persulcatus ticks in Latvia carry several infectious agents, including Borrelia burgdorferi sensu lato (s.l.) and A. phagocytophilum (Ranka et al., [3]; Bormane, [1]). The seroprevalence against Anaplasma phagocytophilum in healthy dogs in Latvia is reported to be $11 \%$ and recently a canine granulocytic anaplasmosis (CGA) case was described (Berzina et al., [4]). Dogs with borreliosis caused by B. burgdorferi and CGA caused by $A$. phagocytophilum present with nonspecific clinical signs lethargy, inappetence, fever, lameness (Beall et al., [5]). Most of the naturally infected dogs resolve these infections without showing clinical signs, however, in cases of

\footnotetext{
* Correspondence: Inese.berzina@gmail.com

Latvia University of Agriculture, Faculty of Veterinary Medicine Preclinical Institute, Pathology department, Kr. Helmana Street 8, Jelgava LV-3004, Latvia
}

double infections disease can be more severe and response to the treatment can be weaker or take longer (Beall et al., [5]). Dogs are recognized as sentinel for human tick-borne diseases, therefore locally generated information on the seroprevalence may be useful for veterinarians and human health specialists alike (Goossens et al., [6]; Hamer et al., [7]).

The aim of this study was to evaluate if seroprevalence against B. burgdorferi s.l. and co-expression of antibodies against B.burgdorferi s.l. and A. phagocytophilum is higher in dogs with clinical suspicion of tick-borne diseases compared to clinicaly healthy dogs.

Two groups of dogs were evaluated - clinically healthy dogs (further in the text - healthy dogs, $n=441$ ) and dogs suspected to have borreliosis and/ or CGA (further in the text - sick dogs, $n=29$ ). The sampling of healthy dogs was planned to cover urban and rural locations and 3 regions depending on the prevalent tick species 
(I. ricinus (IR region, $\mathrm{n}=272$ ), I. persulcatus (IP region, $\mathrm{n}=93$ ), mixed region ( $\mathrm{M}$ region, $\mathrm{n}=76$ ). The number of collected samples was roughly proportional to the density of human population. Detailed explanation on the sample collection and inclusion criteria of dogs have been published previously (Berzina et al., [4]). Two milliliters of venous blood was taken from dogs and serological testing was performed (SNAP 4Dx test, IDEXX Laboratories, Westbrook, Maine, USA) with test that detects antibodies against $A$. phagocytophilum, $B$. burgdorferi sensu lato, Ehrlichia canis and antigen of Dirofilaria immitis. Statistical analysis of the data consisted of the calculation of descriptive statistics and seroprevalence against the pathogens in each of the dog groups (NCSS, 2007, Utah, USA).

In healthy dogs seroprevalence against $B$. burgdorferi s.l. was $2.49 \%(11 / 441)$. More than one third 36\% (4/11) of seropositive dogs co-expressed antibodies against $B$. burgdorferi s.l. and A. phagocytophilum. None of the dogs in the sick dog group expressed antibodies against B. burgdorferi s.l. Seven female and four male dogs, from eight breeds and 1 unknown breed dog were seropositive to $B$. burgdorferi. We did not find significant differences in age, sex or breeds of dogs seropositive against $A$. phagocytophilum versus those seropositive against $B$. burgdorferi s.l. (Berzina et al., [4]).

Co-expression of antibodies was detected only in healthy pet dogs. Three female and one male dog from four different breeds were seropositive against both $B$. burgdorferi s.l. and $A$. phagocytophilum. No statistically significant difference $(\mathrm{p}=0.62)$ was found between the mean ages of the dogs seropositive against $B$. burgdorferi s.l. (5.7 years, $\mathrm{s}=2.9)$ and double seropositive dogs $(6.7$ years, $\mathrm{s}=3.3)$.

Geographical distribution of the seropositive and double seropositive dogs is displayed in the Figure 1. Overall, we can see the trend of higher seropositivity in IR and M regions. Similarly the seropositivity against $A$. phagocytophilum was significantly higher in dogs from IR and $M$ regions (Berzina et al., [4]). However, study by Bormane [1] shows that in Latvia borrelial DNA was equally isolated from $I$. ricinus and I. persulcatus. Both of these tick species have been described as main vectors associated with Lyme disease, I. ricinus in Northern Europe and I. persulcatus in the Eastern Europe (Gray, [8]). To our knowledge, no studies have been conducted to evaluate if these tick species in Latvia have different preferences on feeding on dogs or humans. Additional information on this issue might be added by our ongoing molecular study on the ticks collected from dogs living in IR, IP and M regions of Latvia.

Low seropositivity against borrelia in healthy and lack of seropositivity in sick dogs was unexpected, given the rather high percentage of ticks carrying borrelia and the known incidence of Lyme disease of 31.8 cases per 100,000 humans in Latvia (Ranka et al., [3]; Halperin, [9]). Reported seroprevalence for B. burgdorferi s.l. in dogs in Europe is similarly low 3.9\% in Sweden, 6.5\% in Czech Republic, 1.09\% in France (Egenvall et al., [10]; Pejchalovà et al., [11]; Pantchev et al., [12]) while human disease is common, reaching 69 cases per 100,000 inhabitants in Sweden (Ornastein et al., [13]).

Several authors describe low seropositivity in dogs and even lower number of clinical cases (Beall et al., [5];

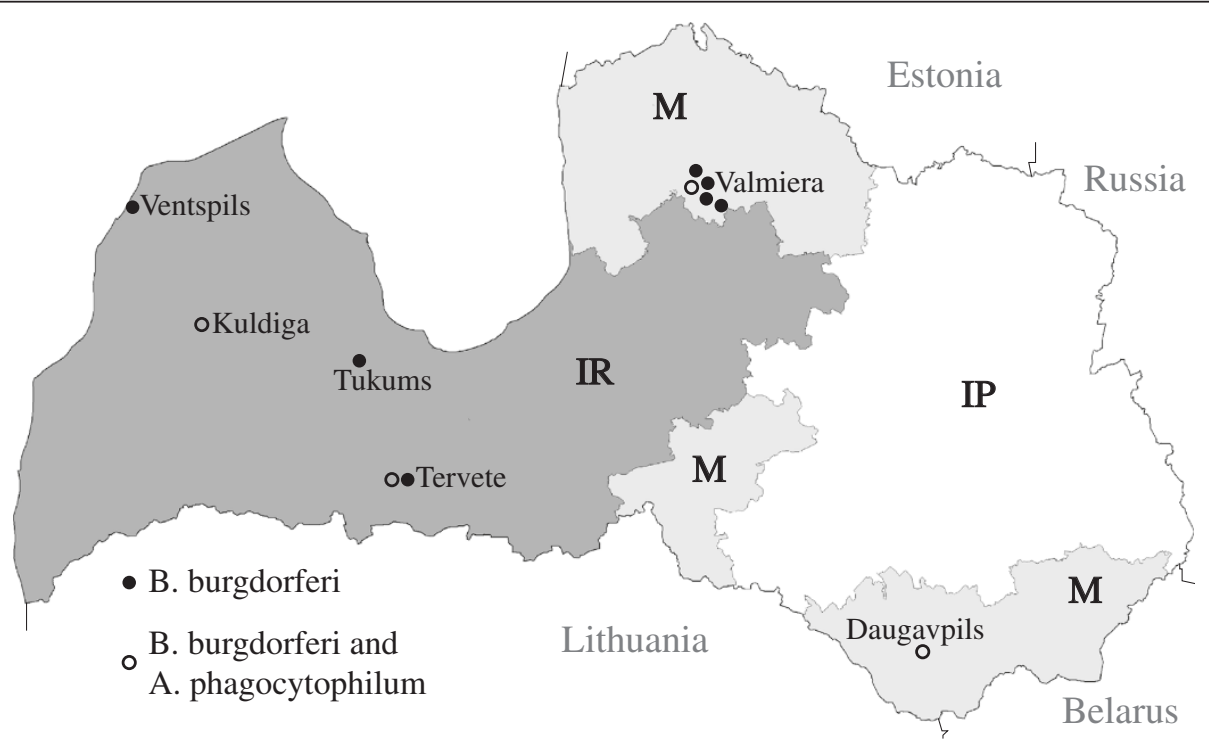

Figure 1 Distribution of dogs seropositive against $B$. burgdorferi sensu lato (B. burgdorferi s.l.) and dogs seropositive against $A$. phagocytophilum and B. burgdorferi s.I. IR - I. ricinus tick habitat, IP - I. persulcatus tick habitat, M - both tick species have been detected. 
Pantchev et al., [12]). This can be partially explained by particularities of the transmission of borrelia to the mammalian host. Transmission can be affected by several factors that include the concentration of the bacteria, length of the feeding of the tick and pathogen species (Kidd et al., [14]). Our results might be affected by several factors -low number of dogs in sick dog group as well as possibility that these dogs might have received antibiotics prior to our testing; the latter might suppress the antibody titer below the detection level of the test (Savic, [15]). Additionally, our results might be affected also by the lower number of investigated dogs from IP and $\mathrm{M}$ regions.

Serological test for B. burgdorferi s.l. used in this study is sensitive and specific ( $94 \%$ and $99.5 \%$ respectively) and detects C6 protein of B. burgdorferi s.l. that is specific to the natural infection, thus vaccinated dogs would be excluded (Beall et al., [5]; Pantchev et al., [12]; Couto et al., [16]).

Although in this study we detected only B. burgdorferi s.l. and A. phagocytophilum antibody co-expression, the possibility of other diseases that are not typical for this geographical location (e.g. ehrlichiosis) still should be kept in mind since pathogens can adapt to be transmitted by a new tick species or ticks can widen their area of habitat or can be "imported" in the country by travelling animals or migrating birds (Cieniuch et al., [17]; Skotarczak et al., [18]; Beall, et al., [5]; Rand et al., [19]).

We conclude that seroprevalence against $B$. burgdorferi s.l. in dogs in Latvia is low, but seropositive dogs frequently co-express antibodies against $A$. phagocytophilum.

\section{Abbreviations \\ B. burgdorferi s.l: B. burgdorferi sensu lato; CGA: Canine granulocytic anaplasmosis; IR region: Region of Latvia, where I. ricinus ticks prevail; IP region: Region of Latvia where I. persulcatus ticks prevail; M region: Region of Latvia where I. ricinus and I.persulcatus ticks have been found.}

\section{Competing interests}

The authors declare that they have no competing interests.

\section{Authors' contributions}

IB performed the sampling, analysis and wrote the draft of this report. IM supervised all stages of the work presented in this report and critically read the report. Both authors read and approved the final manuscript.

\section{Acknowledgement}

This study was funded by European Union and European Social Funds (Agreement No. 2009/0180/1DP/1.1.2.1.2/09/IPIANIAA/017, "Support for Doctoral Studies Program of Latvia University of Agriculture", 04.4-08/EF2. D1.20'). SNAP 4Dx tests were kindly donated by IDEXX laboratories.

Received: 16 November 2012 Accepted: 14 May 2013

Published: 21 May 2013

\section{References}

1. Bormane A: Ixodes ricinus L. and Ixodes persulcatus P.SCh. (Acari: Ixodidae) distribution, significance and molecular epidemiology of transmitted infectious diseases in Latvia. PhD thesis, University of Latvia, Faculty of Biology; 2007.

2. Süss J: Tick-borne encephalitis: Epidemiology, risk areas, and virus strains in Europe and Asia - an overview. TTBDis 2010, 2011(2):2-15.
3. Ranka R, Bormane A, Salmina K, Baumanis V: Identification of three clinically relevant Borrelia burgdorferi sensu lato genospecies by PCRrestriction fragment length polymorphism analysis of 16S-23S ribosomal DNA spacer amplicons. J Clin Microbiol 2004, 42:1444-1449.

4. Berzina I, Capligina V, Bormane A, Pavulina A, Baumanis V, Ranka R, Granta R, Matise I: Association between Anaplasma phagocytophilum seroprevalence in dogs and distribution of Ixodes ricinus and Ixodes persulcatus ticks in Latvia. TTBDis 2013, 4:83-88.

5. Beall MJ, Chandrashekar R, Eberts MD, Cyr KE, Diniz PP, Mainville C, Hegarty BC, Crawford JM, Breitschwerdt EB: Serological and molecular prevalence of Borrelia burgdorferi, Anaplasma phagocytophilum, and Ehrlichia species in dogs from Minnesota. Vector Borne Zoonotic Dis 2008, 8:455-464.

6. Goossens HAT, van den Bogaard AE, Nohlmans ME: Dogs as sentinels for human Lyme borreliosis in the Netherlands. J Clin Microbiol 2001, 39:844-848.

7. Hamer SA, Tsao JI, Walker ED, Mansfield LS, Foster ES, Hickling GJ: Use of tick surveys and serosurveys to evaluate pet dogs as a sentinel species for emerging Lyme disease. Am J Vet Res 2009, 70:49-56.

8. Gray JS: The ecology of ticks transmitting Lyme borreliosis. Exp App/ Acarol 1998, 22:249-258.

9. Halperin JJ: Lyme disease- an evidence-based approach. CAB International Oxfordshire; 2011:103.

10. Egenvall A, Bonnett NB, Gunnarsson A, Hedhammar $\AA$, Shoukri M, Bornstein S, Artursson K: Sero-prevalence of granulocytic Ehrlichia spp. and Borrelia burgdorferi sensu lato in Swedish dogs 1991-94. Scand J Infect Dis 2000, 32:19-25.

11. Pejchalovà K, Žàkovskà A, Fučik K, Schánilec P: Serological confirmation of Borrelia burgdorferi infection in dogs in the Czech Republic. Vet Res Communicat 2006, 30:231-238.

12. Pantchev N, Schaper R, Limousin S, Norden N, Weise M, Lorentzen L: Occurrence of Dirofilaria immitis and tick-borne infections caused by Anaplasma phagocytophilum, Borrelia burgdorferi sensu lato and Ehrlichia canis in domestic dogs in France: results of a countrywide serologic survey. Parasitol Res 2009, 105:101-113.

13. Ornastein K, Berglund J, Nilsson I, Norrby R, Bergström S: Characterization of Lyme borreliosis isolates from patients with erythema migrans and neuroborreliosis in southern Sweeden. J Clin Microbiol 2001, 39:1294-1298.

14. Kidd L, Breitschwerdt EB: Transmission times and prevention of tick-borne diseases in dogs. Compend Contin Educ Pract Vet 2003, 25:742-751.

15. Savic S: Discovering Lyme Disease in Ticks and Dogs in Serbia Detection and Diagnostic Methods, Lyme Disease. In Edited by Ali K. InTech; 2012. http://www.intechopen.com/books/lyme-disease/discovering lyme-disese-in-ticks-and-dogs-in-serbia-detection-and-diagnostic-methods. ISBN 978-953-51-0057-7.

16. Couto CG, Lorentzen L, Beall MJ, Shields J, Bertolone N, Couto Jl, Couto KM, Nash S, Slack J, Kvitko H, Westendorf N, Marin L, lazbik MC, Vicario FC, Sanz $P$, Ruano R: Serological study of selected vector-borne diseases in shelter dogs in Central Spain using point-of-care assays. Vector-borne Zoonotic Dis 2010, 10:885-888.

17. Cieniuch S, Stanczak J, Ruczaj A: The first detection of Babesia EU1 and Babesia canis canis in Ixodes ricinus ticks (Acari, Ixodidae) collected in urban and rural areas in northern Poland. Pol J Microbiol 2009, 58:231-236.

18. Skotarczak B: Canine ehrlichiosis. Ann Agric Environ Med 2003, 10:137-141

19. Rand PW, Lacombe EH, Elias SP, Kahill BK, Lubelczyk CB, Smith RP: Multitarget test for emerging Lyme disease and anaplasmosis in a serosurvey of dogs, Maine, USA. Emerg Infect Dis 2011, 17:899-902.

doi:10.1186/2046-0481-66-9

Cite this article as: Berzina and Matise: Seroprevalence against Borrelia burgdorferi sensu lato and occurence of antibody co-expression with Anaplasma phagocytophilum in dogs in Latvia. Irish Veterinary Journal 2013 66:9. 